-Supporting information-

\title{
Multifunctional Cellular Targeting, Molecular Delivery, and Imaging by Integrated Mesoporous-Silica with Optical Nanocrescent Antenna: MONA
}

Younggeun Park, ${ }^{\dagger} \star \star$ Hyeun Joong Yoon, ${ }^{\S}$ Somin Eunice Lee, ${ }^{\|, \perp, \#, \nabla, \bigcirc}$ and Luke P. Lee ${ }^{\dagger, \bullet, \square, \bigcirc *}$

$\dagger$ Department of Bioengineering and Biomolecular Nanotechnology Center, Berkeley Sensor and Actuator Center and, University of California, Berkeley California, 94720 United States

"Department of Mechanical Engineering, University of Michigan, Ann Arbor Michigan, 48109 United States

${ }^{\S}$ Department of Biomedical Engineering, Michigan Technological University, Houghton Michigan, 49931 United States

"Department of Electrical Engineering and Computer Science, University of Michigan, Ann Arbor Michigan, 48109 United States

${ }^{\perp}$ Department of Biomedical Engineering, , University of Michigan, Ann Arbor, Michigan 48109, United States

\#Applied Physics, , University of Michigan, Ann Arbor, Michigan 48109, United States

${ }^{\nabla}$ Macromolecular Science and Engineering, University of Michigan, Ann Arbor, Michigan 48109, United States

○Biointerfaces Institute, University of Michigan, Ann Arbor Michigan, 48109 United States

-Department of Electrical Engineering and Computer Science, University of California, Berkeley California, 94720 United States

$\square$ Harvard Institute of Medicine, Harvard Medical School, Harvard University, and Department of Medicine, Brigham and Women's Hospital, Boston, MA, 02115 United States

${ }^{\circ}$ Institute of Quantum Biophysics, Department of Biophysics, Sungkyunkwan University, Suwon, Korea

${ }^{*}$ Corresponding author: 1plee@bwh.harvard.edu 


\section{List of figures}

\section{Materials and experimental section}

Figure S1. Scattering spectra normalized $\left(I_{\text {scat }}\right)$ of NC filled with different cavity materials; air, water, and mesoporous silica nanosphere (MSN) at $\left(D_{\text {cavity }}=40\right.$ and $\left.D_{A u}=50 \mathrm{~nm}\right)$.

Figure S2. Flow cytometric sorting of MCF-7 cells with MONA conjugated EpCAM and isotope.

Figure S3. Temperature profile in MONA at $650 \mathrm{~nm}($ scale bar $=50 \mathrm{~nm})$.

Figure S4. Pore structure characterization. a) Nitrogen adsorption and b) pore size distribution of MONA and MONA-DOX.

Figure S5. Particle dispersion in the various mediums. Spectra of MONA colloidal particles as a function of particle density a) PBS and b) Dulbecco's Modified Eagle Medium (DMEM) $+10 \%$ Fetal Bovine Serum (FBS).

Figure S6. DOX release from MONA; A, Cumulative DOX release dynamics from MONA-DOX $\left(d_{M S N}=0.1 \mathrm{mg} / \mathrm{mL}\right.$ and $\left.C_{D O X}=1 \mu \mathrm{M}\right)$ at $\mathrm{pH}=6.25$. B, Correlation curve between DOX loading concentration and loaded DOX concentration in $\mathrm{MSN}\left(d_{M S N}=0.1 \mathrm{mg} / \mathrm{mL}\right)$ at $\mathrm{pH}=7.4$.

Figure S7. Characterization of MONA activation. (a) Normalized fluorescence versus time plots comparing photothermal release as different power densities. (b) Normalized fluorescence versus time plots comparing photothermal release for a laser at the peak optical absorption $(\lambda=$ $650 \mathrm{~nm})$ and a laser outside the peak optical absorption $(\lambda=530 \mathrm{~nm})$.

Figure S8. Cell viability test. (A) Cell viability of MCF-7 cells treated with MONA-DOX as a function of particle density at $C_{D O X}=1 \mu \mathrm{M}$. (B) Cell viability of MCF-7 cells incubated with MONA-DOX for $72 \mathrm{~h}$, as a function of lorded DOX concentration.

Figure S9. Comparison test. Linear regression between MTT assay and PRET data for the same samples treated with DOX-MONA ranging from 0.1 to $5 \mathrm{uM}\left(\mathrm{R}^{2}=9744\right)$.

Figure S10. Dynamic intracellular PRET data. (A) PRET signals as a function of time; $t_{1}=2$, $t_{2}=4, t_{3}=8, t_{4}=16$, and $t_{5}=24 \mathrm{~h}$. (B) PRET data of control sample as a function of time; $t_{1}=2, t_{2}$ $=4, t_{3}=8, t_{4}=16$, and $t_{5}=24 \mathrm{~h}$.

Figure S11. The spectral selectivity of MONA allowing a potential in animal and tissue level study. a) Schematic showing the change in the optical skin-depth at different wavelength of incident light in the tissue geometry, b) Optical windows in biological tissues. These plots of effective attenuation coefficient versus wavelength show that absorption and scattering from oxygenated blood, deoxygenated blood, skin and fatty tissue is lowest in either the first or second near-infrared window. c) schematic of MONA structures with edge-edge distances of i) 40, ii) 25 , iii) 20 , iv) $10 \mathrm{~nm}$, v) $5 \mathrm{~nm}$, and vi0 $2 \mathrm{~nm}$. d) Extinction spectra of MONA structures estimated with edge-edge distances of i) 40 , ii) 25 , iii) 20 , iv) $10 \mathrm{~nm}$, v) $5 \mathrm{~nm}$, and vi) $2 \mathrm{~nm}$. 


\section{Experimental section}

Synthesis of mesoporous silica nanosphere, MSN: Cetyltrimethylammonium bromide (CTAB) $(1.17 \mathrm{~g})$ was dissolved in a solution containing water $(180 \mathrm{~mL})$ and ethylene glycol EG $(30 \mathrm{~mL})$ in an aqueous solution of ammonia $(7.2 \mathrm{~mL}, 25$ percent $)$. TEOS $(1.43 \mathrm{~mL})$ and APS $(0.264 \mathrm{~mL})$ were rapidly added to the mixture after vigorous stirring for about $30 \mathrm{~min}$ at $323 \mathrm{~K}$. In the solution, the final molar composition was 1 TEOS: 0.18 APS: 0.50 CTAB: $13.2 \mathrm{NH}_{3}: 84$ EG: $1561.1 \mathrm{H}_{2} \mathrm{O}$. The resulting mixture was stirred at $50{ }^{\circ} \mathrm{C}$ for another $2 \mathrm{~h}$ and then statically left for $20 \mathrm{~h}$ at the same temperature. We collected MSN samples with subsequent washing and re-dispersing steps by centrifugation at $20,000 \mathrm{rpm}$ for $20 \mathrm{~min}$. In a centrifuge tube, the synthesized

MSNs were dried at $60^{\circ} \mathrm{C}$. Then the MSNs $(1 \mathrm{~g})$ and Ammonium nitrate (NH4NO3) $(0.3 \mathrm{~g})$ were dissolved in ethanol $(40 \mathrm{~mL})$ and heated to extract the surfactant at $60{ }^{\circ} \mathrm{C}$. We obtained a final MSNs ample via the next centrifugation.

MSN functionalization: We first prepared a mixture of BPS $(40 \mu \mathrm{L})$ and MSN colloidal suspension (10 mg per $100 \mathrm{~mL}$ of ethanol) and refluxed it to functionalize the prepared MSN with 3bromopropyltriethoxysilane (BPS). We twice performed centrifugation and washing steps with ethanol after $3 \mathrm{~h}$. Then, in DI water, we dispersed the bromine-functionalized MSN particles. We stirred it for $1 \mathrm{~h}$ after adding hydrazine $(0.2 \%$ in $100 \mathrm{~mL}$ of DI water). Finally, we obtained a functionalized MSN sample after performing the centrifugation and washing steps three times.

Integration of AuNC nanoantenna with MSN: We then used e-beam deposition method to integrate AuNC with MSN. Firstly, by rinsing it with toluene, acetone, ethanol, and DI water, we prepared a clean $\mathrm{SiO}_{2}$ substrate. After air-drying, $\mathrm{O}_{2}$ plasma treatment of the surface of the $\mathrm{SiO}_{2}$ substrate was performed for $2 \mathrm{~min}$ at $\mathrm{P}=18 \mathrm{~W}$ to create hydroxyl groups on it (COVANCE 1-MP, Femto). 
Then the MSN colloidal solution was dropped and cast on the $\mathrm{SiO} 2$ substrate (0.1 percent in DI water). We dried the substrate in a clean-zone hood overnight in order to evaporate the solvent on the substrate gently. We rinsed the substrate gently to remove extra particles and dust on the substrate after drying the solution, using DI water, and dried the substrate again. With the substrate, we conducted $\mathrm{Au}$ deposition in different thicknesses using the E-beam evaporation method (Angstrom Engineering Evovac Evaporator). During the Au deposition (Deposition rate $=0.02 \mathrm{~nm}$ / sec), we put the substrate above the Au sources with a certain tilt angle $\left(\sim 60^{\circ}\right)$ at a constant rotating speed $(100 \mathrm{rpm})$. We isolated the Au coated MSNs from the glass substrate by ultrasonic treatment for $2 \mathrm{~h}$ after completion of the deposition. The Au coated MSNs were then gathered by centrifugation ( $\sim 10,000 \mathrm{rpm}, 10 \mathrm{~min})$ and suspended with ultrasonic treatment in DI water. The sample was then centrifuged and washed three to four times in water.

Characterization of MONA: Nitrogen adsorption-desorption measurements were performed using an ASAP 2020 sorptometer at 77K to characterize pore size, surface area, and volume. The BJH (Barrett-Joyner-Halenda) method underestimates the characteristics of ink-bottle or spherical pores for pore sizes above $7 \mathrm{~nm}$, while the BdB-FHH (Broekhoff-de Boer-Frenkel-Halsey-Hill) model is more accurate in ranges over $5 \mathrm{~nm}$ than the $\mathrm{BJH}$ model. And thus pore size distribution (PSD) and pore volumes were measured by the BdB-FHH method, and specific surface areas were calculated using the BET method. We obtained TEM images using a JEM-200CX electron microscope operating at an accelerating voltage of $200 \mathrm{keV}$ to characterize the shapes and geometries of the MONA. SEM images with a FE-SEM (Hitachi SU8000) were acquired at a $10 \mathrm{KV}$ accelerating voltage for morphology analysis.

DOX loading into porous structure of MONA: To load DOX into MONA, we dispersed the prepared MONA in $1 \mathrm{~mL}$ of methanolic doxorubicin solution $(5 \mathrm{mg} / \mathrm{mL})$. We stirred it overnight 
in the dark state to induce weak bonding between MONA and DOX in the pore and removed unloaded DOX by centrifugation and methanol and $\mathrm{pH} 7.4$ buffer washing steps. After collecting the DOX loaded MONA (MONA-DOX), to estimate the amount of loaded DOX into the MSN, we measured UV absorption $(\lambda=480 \mathrm{~nm})$.

DOX release test: MONA-DOX particles $(1 \mathrm{mg})$ were dispersed in a $1 \mathrm{~mL}$ buffer at different $\mathrm{pH}$ conditions from 7.4 to $6.5(10 \mathrm{mM}$ phosphate buffer $(\mathrm{pH} 7.4-6.0)$ and $10 \mathrm{mM}$ acetate buffer $(\mathrm{pH}$ $5.5-4.0))$ to test the DOX release. The solution of the particle mixture was centrifuged at intervals of $1 \mathrm{~h}$. The same volume of fresh buffers was added back to the residual mixture to wash the sample. By measuring the UV absorbance intensity of DOX in supernatant solution after centrifugation, we monitored drug release. In addition, the light-responsive properties were evaluated and the MONA-DOX particle (1 mg) was dispersed individually in $1 \mathrm{~mL}$ buffer under light on $\left(\lambda=650 \mathrm{~nm}=\sim 5 \mathrm{~mW} / \mathrm{cm}^{2}\right.$ for $\left.10 \mathrm{~min}\right)$ and dark conditions at $10 \mathrm{mM}$ phosphate buffer. The particle mixture solution was centrifuged after incubation at intervals of $1 \mathrm{~h}$. The same volume of fresh buffers was added back to the residual mixture to wash the sample. We monitored the release of light-dependent drugs by measuring the intensity of DOX after centrifugation in the supernatant solution.

In vitro cell viability (MTT) test: MCF-7 cells were seeded in a 96-well plate at a density of $10^{5}$ cells per well and cultured in $5 \% \mathrm{CO}_{2}$ at $37{ }^{\circ} \mathrm{C}$ for $72 \mathrm{~h}$ to test the cytotoxicity of MONA-DOX and MONA. We then added MONA-DOX and MONA into the medium, and for $72 \mathrm{~h}$, the cells were incubated in $5 \% \mathrm{CO}_{2}$ at $37{ }^{\circ} \mathrm{C}$. At the end of the incubation, $100 \mu \mathrm{L}$ of MTT solution (diluted in a culture media with a final concentration of $0.1 \mathrm{mg} / \mathrm{mL}$ ) was added and incubated for another $4 \mathrm{~h}$. The medium was then replaced with $100 \mu \mathrm{L}$ of dimethyl sulfoxide (DMSO) per well, and the 
absorbance was monitored using a microplate reader (Bio-TekELx800) at the wavelength of 595 $\mathrm{nm}$. The cytotoxicity was expressed as the percentage of cell viability compared to untreated control cells. The optical density (OD) of the sample was measured at $570 \mathrm{~nm}$ with a microplate reader. The cytotoxicity (eq. 1) was calculated as follows:

$$
\text { Cytotoxicity }=(A-B) / \mathrm{A} \times 100 \%
$$

,where $A$ is the absorbance of the cells incubated with the culture medium and $B$ is the absorbance of the cells incubated with the nanoparticles or the free molecules.

Antibody functionalization on MONA: At first, we centrifuged a solution suspending MONA three times at 5,000 rpm for $10 \mathrm{~min}$ and washed in D.I. water. Subsequently, we functionalized the MONA with thiolated alkane 10-Carboxy-1-decanethiol $\left(\mathrm{HS}-\left(\mathrm{CH}_{2}\right) 10-\mathrm{COOH}\right)$ using a selfassembly method (SAM). In this process, MONA colloidal solution was first incubated in $1 \mathrm{mM}$ of thiolated alkane 10-Carboxy-1-decanethiol (HS- $\left.\left(\mathrm{CH}_{2}\right)_{10}-\mathrm{COOH}\right)$ overnight. Then, the antibody was linked to the $-\mathrm{COOH}$ functional group formed on the AuNP surface by means of NeutrAvidin chemistry. Here, we first washed the - $\mathrm{COOH}$ functionalized MONA and loaded these MONA into a mixture of NeutrAvidin $(50 \mathrm{mg} / \mathrm{mL})$ in a $\mathrm{N}-\gamma$-maleimidobutyryl-oxysuccinimide ester (GMBS) solution to activate the MONA surface. We loaded a solution of primary biotinylated EpCAM antibody diluted from 100 to $10 \mu \mathrm{g} / \mathrm{mL}$ in 1x PBS into the micro-tube holding the solution of the MONA functionalized above and incubated it for $60 \mathrm{~min}$. To suppress fouling of the MONA surface due to non-specific binding, we treated the prepared antibody-MONA conjugates with $1 \%$ BSA in 1x PBS in blocking buffer and incubated the whole system for 20 min. Before the test, the antibody-MONA particles were thoroughly washed three times to remove any excessive solutions or molecules using $20 \mu \mathrm{L}$ of $1 \times$ PBS. At the last stage, we compared the extinction spectrum of the 
MONA colloidal solution before and after the functionalization using a UV-VIS spectrometer (Agilent 8453 G1103A Spectrophotometer).

Fluorescence microscopy images of cell interaction with biofunctionalized MONA: To identify the conjugated anti-EpCAM of MONA on the cell membrane by fluorescence microscopy, we first labelled a fluorescent secondary antibody. Then, we incubated MCF-7 cells $\left(1 \times 10^{6}\right.$ cell $\left./ \mathrm{mL}\right) 50$ $\mu \mathrm{L}$ of anti-EpCAM-MONA, as a prepared solution. The labeled cells were centrifuged, washed twice after incubation ( $30 \mathrm{~min}$ at $\mathrm{T}=25^{\circ} \mathrm{C}$, with gentle agitation) to remove the excess of antiEpCAM that was functionalized on anti-EpCAM-MONA and re-dispersed in a buffer. After centrifugal washing, the pellet was re-suspended and incubated with fluorescein isothiocyanate (FITC)-conjugated secondary anti-rabbit antibodies used as a fluorescence analysis mark. Controls were carried out with MONA without anti-EpCAM, as well as with MONA combined with various types of antibodies which proved to be non-specific to MCF-7 cells.

Flow cytometry test: For flow cytometry analysis was performed on MCF-7 cells to validate the targeting function of MONA. The cells are washed three times with 1X PBS, detached from the cell culture dish using 1m M EDTA for 10 minutes, collected using centrifugation (1800 rpm, 4 minutes), and re-suspended in PBS containing 2\% fetal bovine serum (16000-036, Invitrogen Corporation) and $0.1 \%$ sodium azide. For targeting, $1 \times 10^{6}$ cells were resuspend in $200 \mathrm{uL}$ of $3 \%$ BSA in PBS containing MONA which has a fluorescence tag (Alex 488) and incubated in the dark over ice for 30 minutes. Cells were then analyzed on a flow cytometry (ZE5 cell analyzer, BioRad). A total of 10,000 events were collected per sample and analyzed using FCS express software.

In cellulo apoptosis study and dark field/PRET measurement: For the intracellular molecular delivery, $100 \mu \mathrm{L}$ of conjugated MONA particles $(1 \mathrm{mg} / 1 \mathrm{~mL})$ were internalized into MCF-7 cells 
which are placed in a petri dish. The cells are removed from the petri dish after 30 minutes, and are washed three times with 1 PBS. Afterwards, the cell is mounted on a microscope slide. A PDMS micro chamber was used to form a well around the coverslip on the microscope slide. This well was packed with media then. The microscopy system consisted of an inverted microscope (Olympus IX73) equipped with a dark-field condenser (1.2 -1.4 numerical aperture) and a white light source (Xenon Arc Lamp) to acquire the PRET signal and images. The cell culture plate was mounted on the microscope for PRET measurement from live cells, and the spectra were obtained with the same technique from the intracellular probes. Using the focused laser $(=650 \mathrm{~nm})$ at 5 $\mathrm{mW} / \mathrm{cm}^{2}$ for 10 minutes for intracellular molecular transmission, the internalized MONA particles were illuminated. Then, using a digital camera, the dark images of the treated cell and MONA were acquired (Q-color3, Olympus). A monochromator (300 mm focal length and 300 grooves per mm, Acton Research) with a cooled spectrograph CCD camera was used to gather scattering spectra from the samples at different locations (Roper Scientific). In front of the monochromator, we set up a $2 \mu \mathrm{m}$-wide aperture to hold only a single probe in the region of interest.

Spectral analysis: We obtained the baseline and normalized signals of the acquired scattering spectrum with the equation, $I=\left(I_{A u_{-} \text {Nano antenna in cell }}-I_{\text {baseline }}\right) /\left(I_{A u_{-} \text {Antenna }}-I_{\text {baseline }}\right)$, where $I_{A u_{-} N a n o}$ antenna in cell and $I_{A u_{-} \text {Nano antenna }}$ in medium are an intensity obtained from the Au nano antenna structure inside a cell and outside a cell (in medium), respectively. We obtained $I_{\text {baseline }}$ in the absence of any light sources. The acquired spectrum was processed according to the adjacent averaged method. For quantification for released intracellular $c y t c$ level, we calculated changes in quenching dip intensities between $520-530 \mathrm{~nm}$ were by the subtraction with its initial intensity, $\Delta I=\left|\left(I_{t 0}-I_{t}\right) / I_{t 0}\right|$, where $I_{t 0}$ and $I_{t}$ are intensities at $t=0$ and $\mathrm{n}$, respectively. 
EpCAM receptor targeting: MONA suspension $(10 \mu \mathrm{L}, 0.1 \mathrm{mg} / \mathrm{mL})$ was added into Phosphate buffered saline (PBS) solution $(10 \mu \mathrm{L})$ containing antibodies $(2.5 \mu \mathrm{g}$, anti-EpCAM, Becton Dickinson, NJ, USA). After incubating overnight at $4{ }^{\circ} \mathrm{C}$, the mixture was then centrifuged and the MONA were suspended in PBS $(100 \mu \mathrm{L})$. The functionalized MONA were then loaded onto cultured breast cancer cells (MCF-7 cell line) for $30 \mathrm{~min}$ at $37^{\circ} \mathrm{C}$, followed by washing cells with PBS $1 \times$ with sodium azide $(0.1 \%)$ thoroughly to remove the unbound MONA. After dying the cells with Hoechst 33342, the cells were fixed with paraformaldehyde (1\%) for imaging. As a control, same targeting experiment using functionalized the MONA with isotype antibodies (mouse Ig fluorescence controls) were repeated the procedure described above. To confirm that the anti-EpCAM functionalized MONA has no attachment to cells that do not over-express EpCAM, other cells were used following the procedure described above.

Near-field electromagnetic radiation calculation: The dimensions of the AuNC (Exterior diameter $\left(d_{e x t}\right)=60 \mathrm{~nm}$, Interior diameter $\left(d_{i n}\right)=20 \mathrm{~nm}$, and Asymmetric gap distance between exterior and interior diameter $\left.\left(l_{\text {ext-int }}\right)=25 \mathrm{~nm}\right)$ were chosen based on the SEM images as shown in figure 2 . Because the AuNC consists of sharp and complex geometry, the FEA based on adaptive meshes producing capability enabled obtaining the near field near-field electromagnetic radiation. To estimate near-field electromagnetic radiation around an NC structure, using a finite element analysis method (FEA, COMSOL Multiphysics software), we solved the Helmholtz wave equation $\left(\nabla \times\left(\mu_{r}^{-l} \nabla \times E\right)-\mathrm{k}_{0}^{2}\left(\varepsilon_{r}-j \sigma \omega \varepsilon_{0}\right) E=0\right)$. We introduced hybrid mesh structures to adopt its round and sharp geometry of AuNC shape. We assumed that relative permeability $\left(\mu_{r}\right)$ and complex permittivity $\left(\varepsilon_{r}\right)$ of Au were $\mu_{r}=1$ and $\varepsilon_{r}=f(\lambda)$, respectively. Regarding the outer boundary condition, we set perfect absorption to minimize spurious reflections with a perfectly matched layer and an integration layer in the concentric space. 
Multiphysics simulation of MONA: To estimated photothermal effect, we used the same geometry that was used in the Near-field electromagnetic radiation calculation, and we simulated a thermal energy conversion spectrum ( $q$ ) of the AuNC antenna. The spot of the highest $q$ in the MONA is exactly matched to that of the strongest cross-section of scattering. Subsequently, we calculated near-field distribution of the photothermal parameters $\left(|E|, E_{x}, q\right.$ and $\left.T\right)$. The amount of photon-tophonon conversion is a function of the intensity of the electric field (eq. 2).

$$
q(\mathrm{r})=\frac{n^{2} \omega}{2} \cdot \operatorname{Im}\left(\epsilon_{\omega}\right) \cdot|\boldsymbol{E}(\boldsymbol{r})|^{2}
$$

, where $q$ is thermal energy, $n$ is refractive index, $\omega$ is frequency, $l m$ is imaginary part of dielectric properties, $E$ is electric-field. The focused $E$ on the MONA is efficiently converted into $q$. Local temperature profile of MONA was calculated by using heat trasnfer equation (eq. 3).

$$
\kappa \nabla^{2} \mathrm{~T}(\mathbf{r})=-\mathrm{q}(\mathbf{r})
$$

, where $\mathrm{K}$ is thermal diffusion coeffcient and $\mathrm{T}$ is local temperature. Photothermal effect mostly occurs at the edge tip of the MONA. The photothemal enegy is quickly diffused along with the AuNC antenna curve structure in the MONA. 


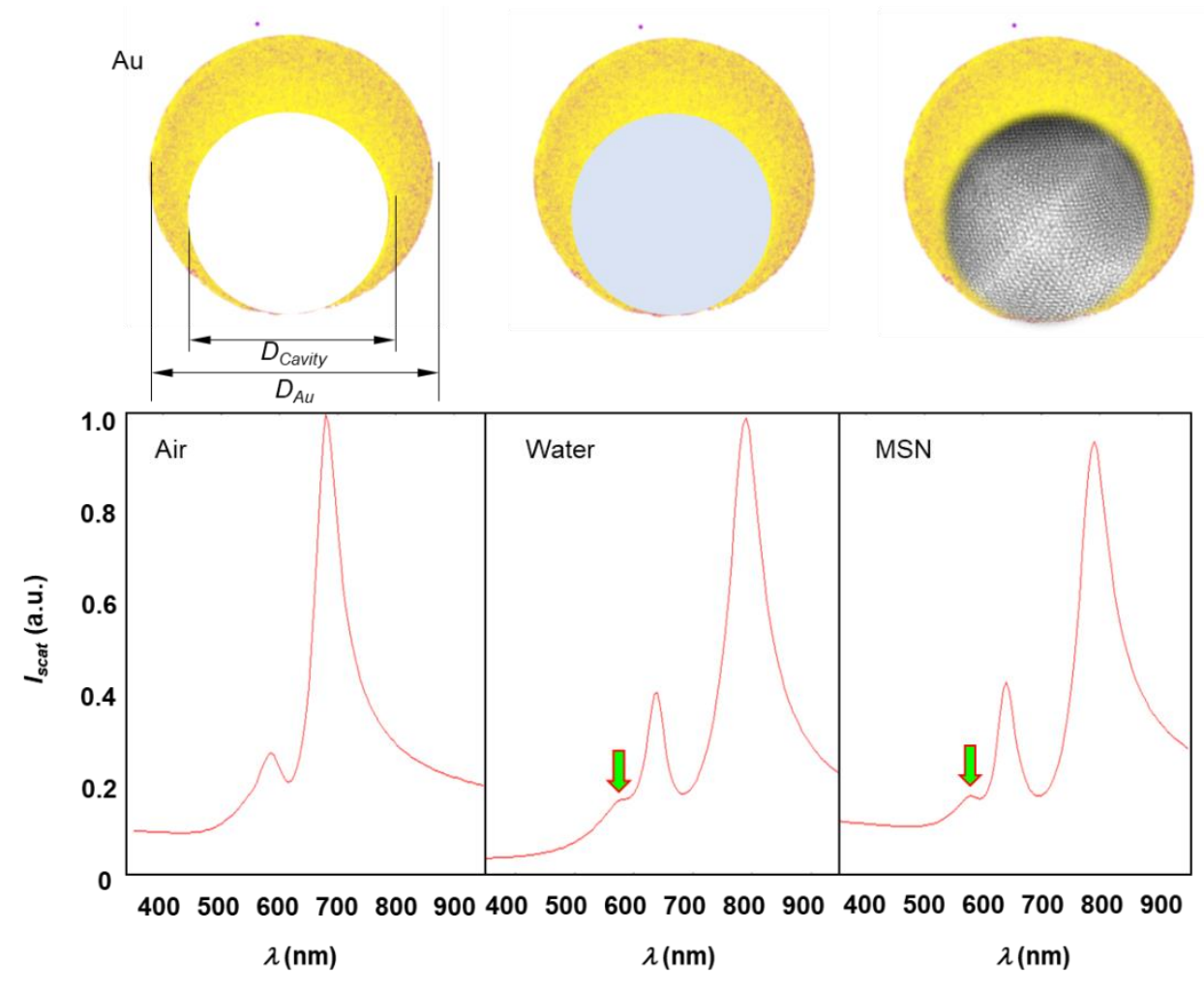

Figure S1. Scattering spectra normalized $\left(I_{\text {scat }}\right)$ of MONAs filled with different cavity materials; air, water, and mesoporous silica nanosphere at $\left(D_{\text {cavity }}=40\right.$ and $\left.D_{A u}=50 \mathrm{~nm}\right)$. 
a

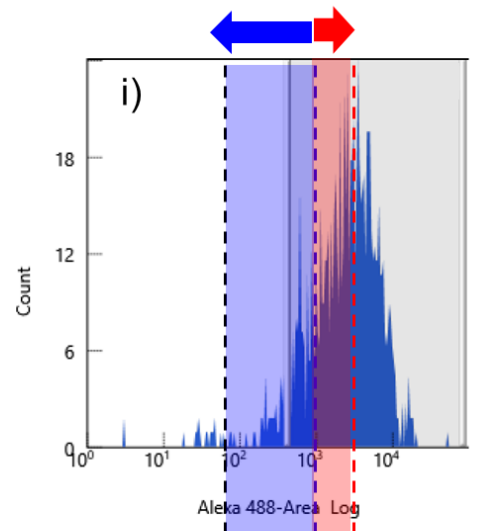

b

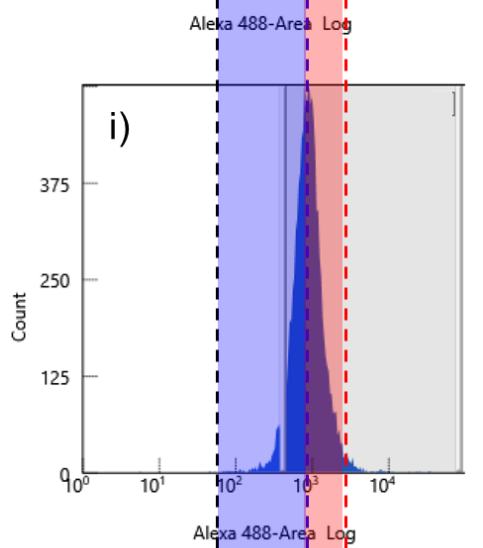

C

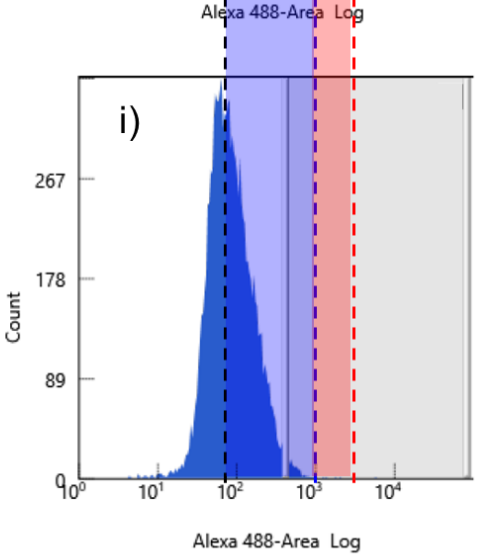

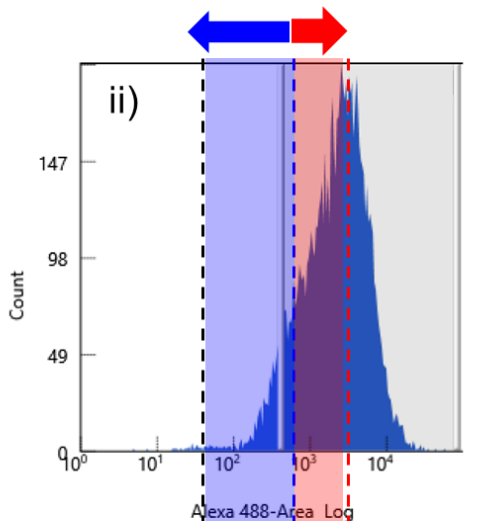
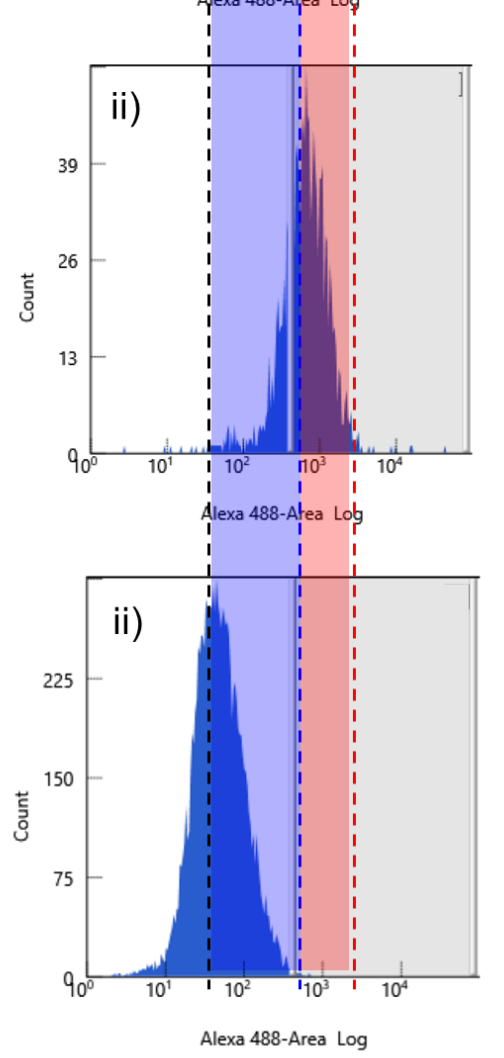

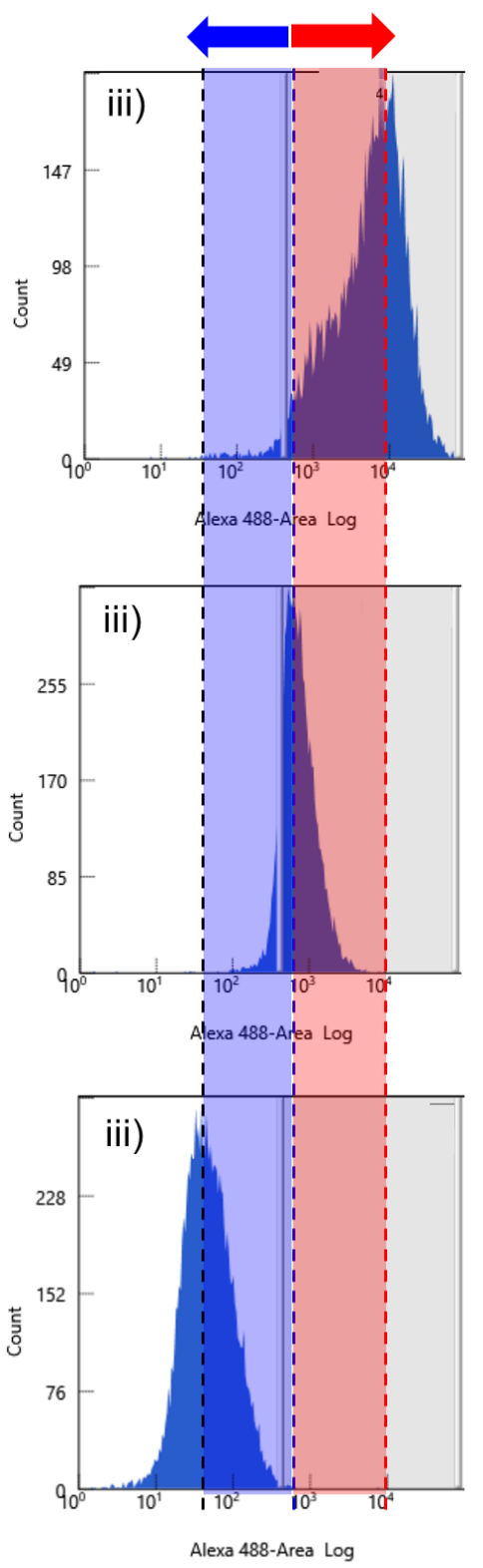

Alexa 488-Area Log

Figure S2. Flow cytometric sorting of MCF-7 cells with MONA conjugated. a) EpCAM and b) Isotype Control (Mouse IgG2a kappa) with MCF-7 cells, and c) EpCAM with Hs-578T cells at different particle density; i) $1 \times 10^{-2}$, ii) $5 \times 10^{-2}$, and iii) $1 \times 10^{-1} \mathrm{mg} / \mathrm{mL}$. 


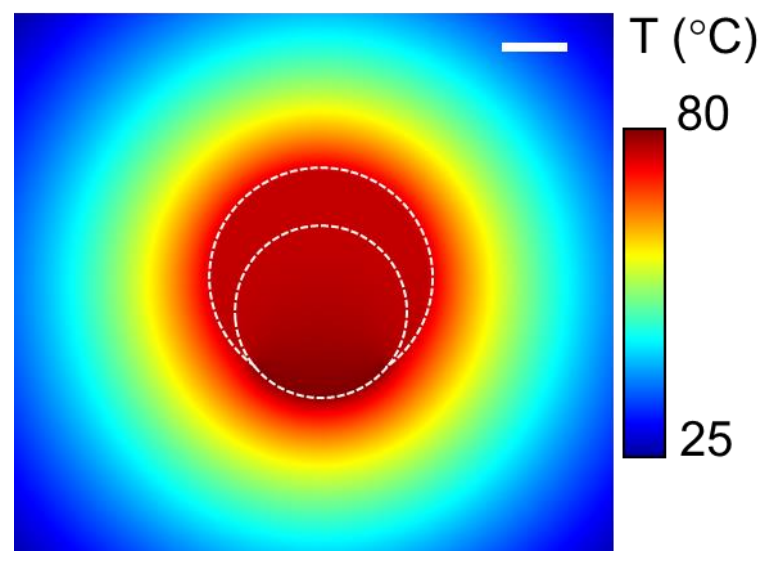

Figure S3. Temperature profile in MONA at $650 \mathrm{~nm}$ with $6.5 \mathrm{~mW} / \mathrm{mm}^{2}$ (scale bar $\left.=25 \mathrm{~nm}\right)$. 
a

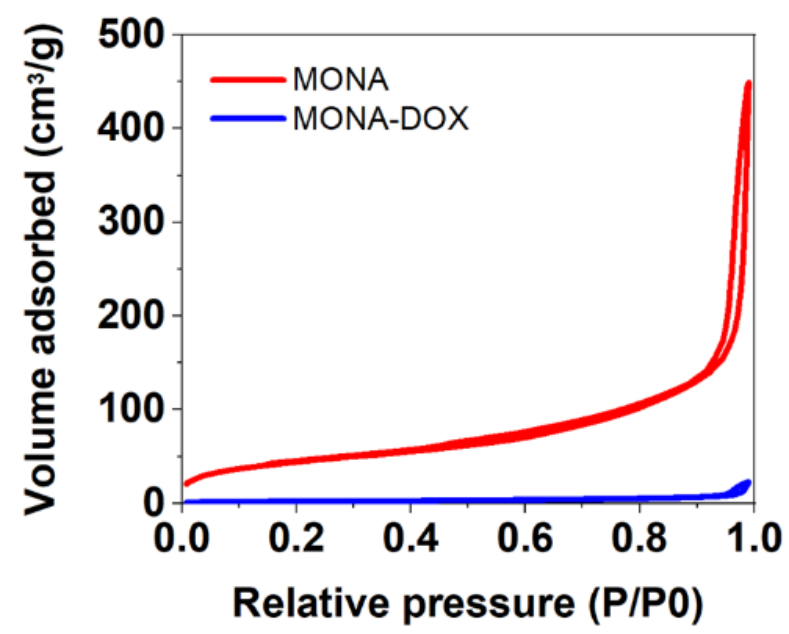

b

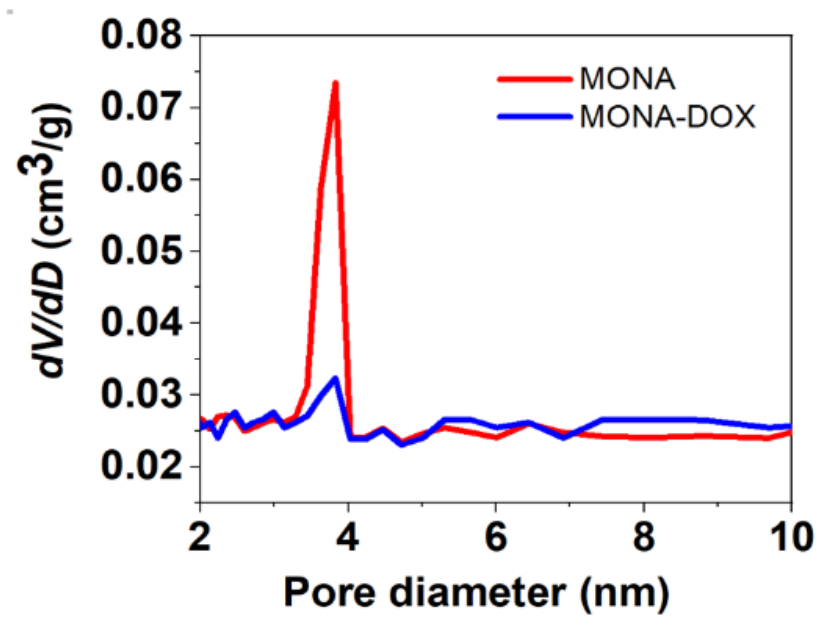

Figure S4. Pore structure characterization. a) Nitrogen adsorption and b) pore size distribution of MONA and MONA-DOX $\left(\mathrm{C}_{\mathrm{DOX}}=0.1 \mathrm{mg} / \mathrm{mL}\right)$. 


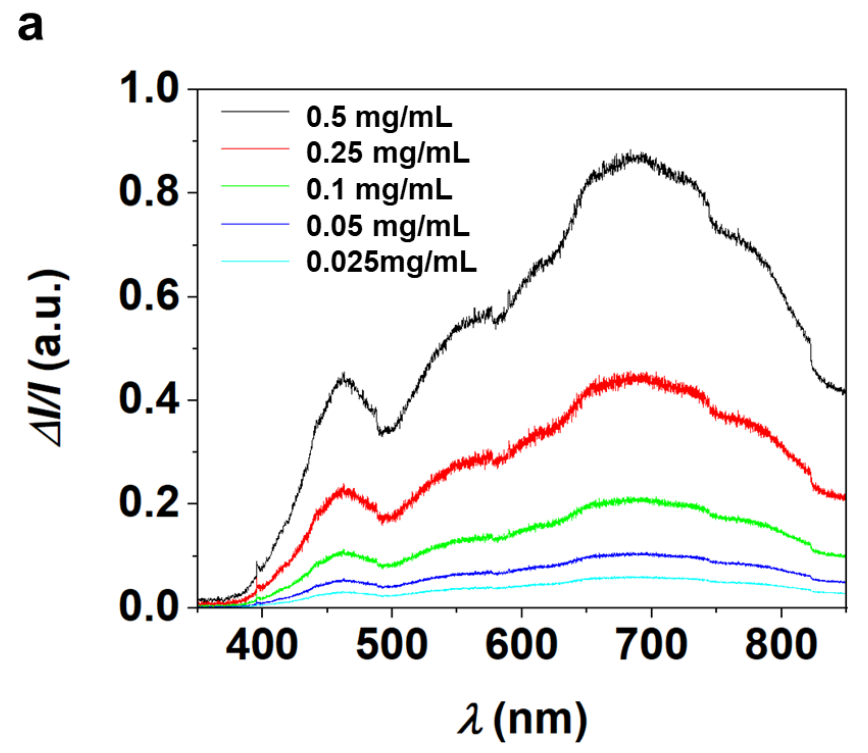

b

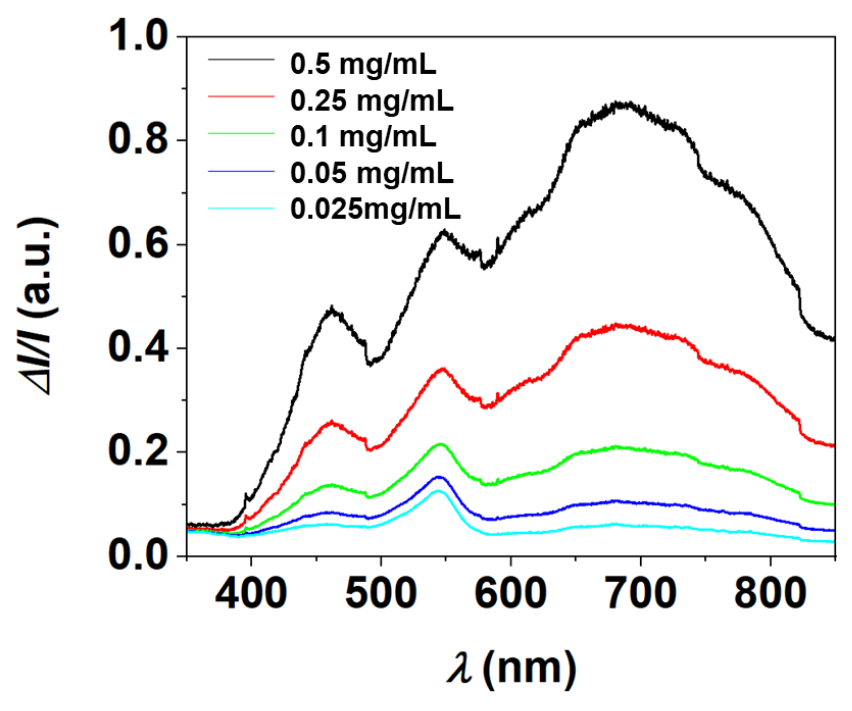

Figure S5. Spectra of MONA colloidal particles as a function of particle density in a) PBS and b) Dulbecco's Modified Eagle Medium (DMEM) + 10\% Fetal Bovine Serum (FBS). 
a

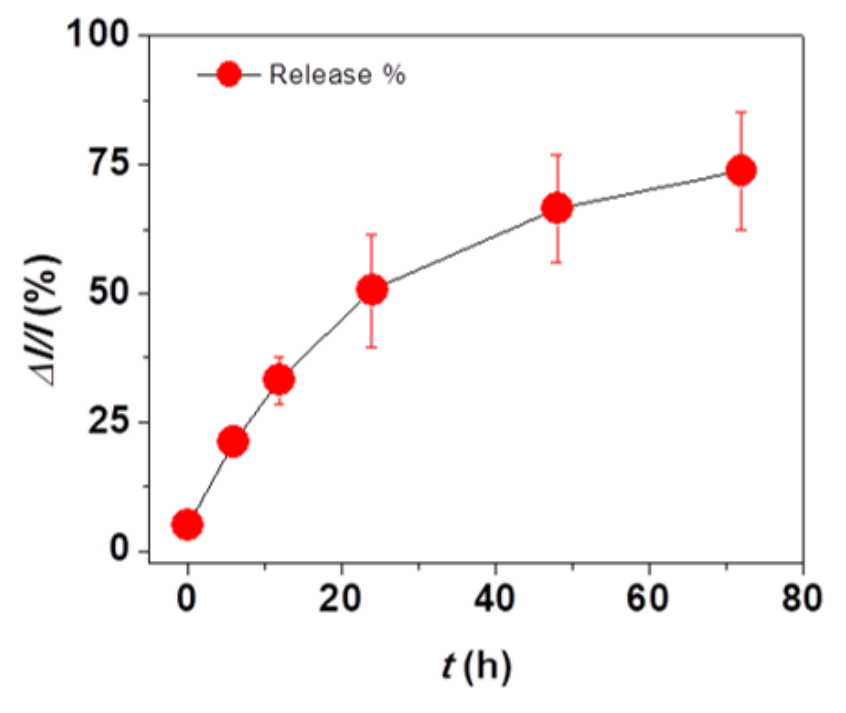

b

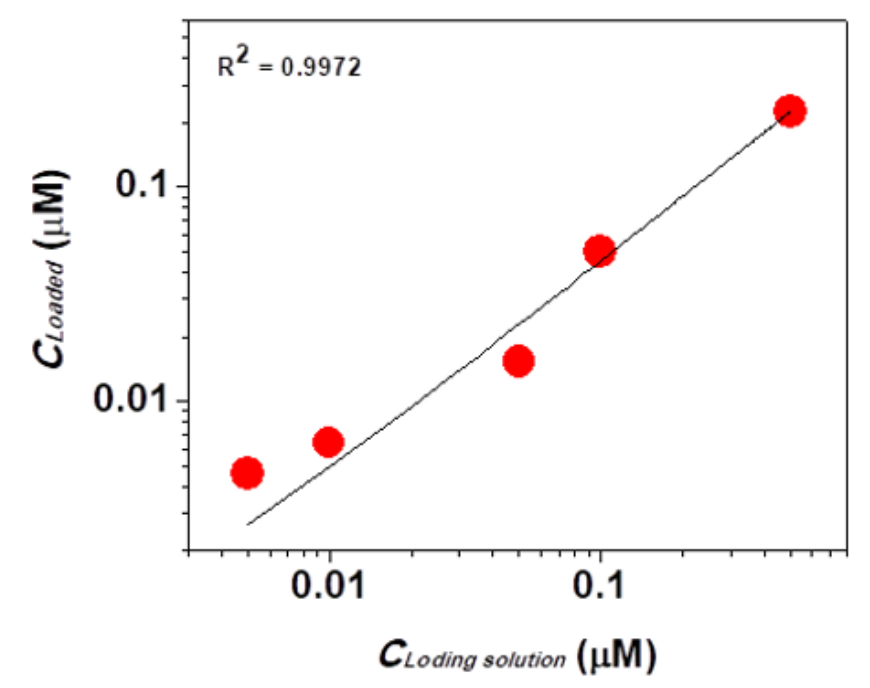

Figure S6. DOX release from MONA; a, Cumulative DOX release dynamics from MONA-DOX $\left(d_{M S N}=0.1 \mathrm{mg} / \mathrm{mL}\right.$ and $\left.C_{D O X}=1 \mu \mathrm{M}\right)$ at $\mathrm{pH}=6.25 . \mathrm{b}$, Correlation curve between DOX loading concentration and loaded DOX concentration in $\operatorname{MSN}\left(d_{M S N}=0.1 \mathrm{mg} / \mathrm{mL}\right)$ at $\mathrm{pH}=7.4$. 


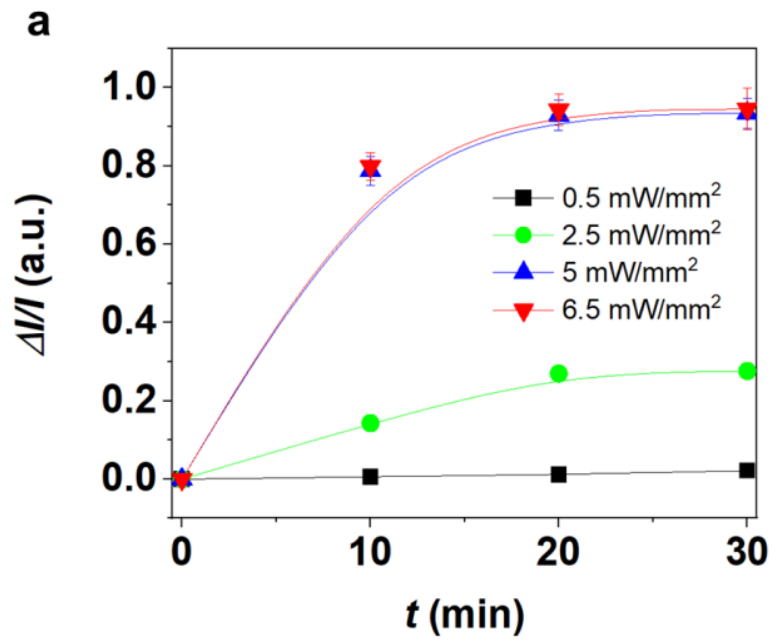

b

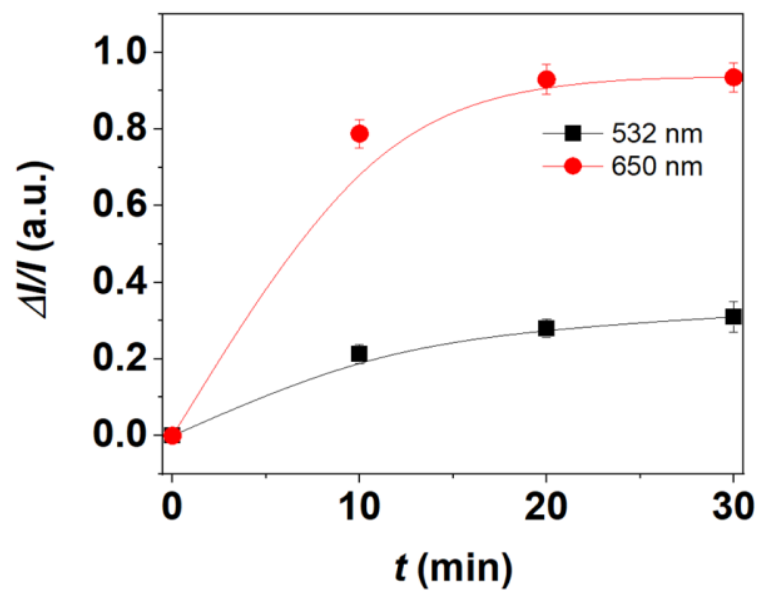

Figure S7. Characterization of MONA activation. (a) Normalized fluorescence versus time plots comparing photothermal release as different power densities. (b) Normalized fluorescence versus time plots comparing photothermal release for a laser at the peak optical absorption (650 $\mathrm{nm})$ and a laser outside the peak optical absorption $(530 \mathrm{~nm})$. 


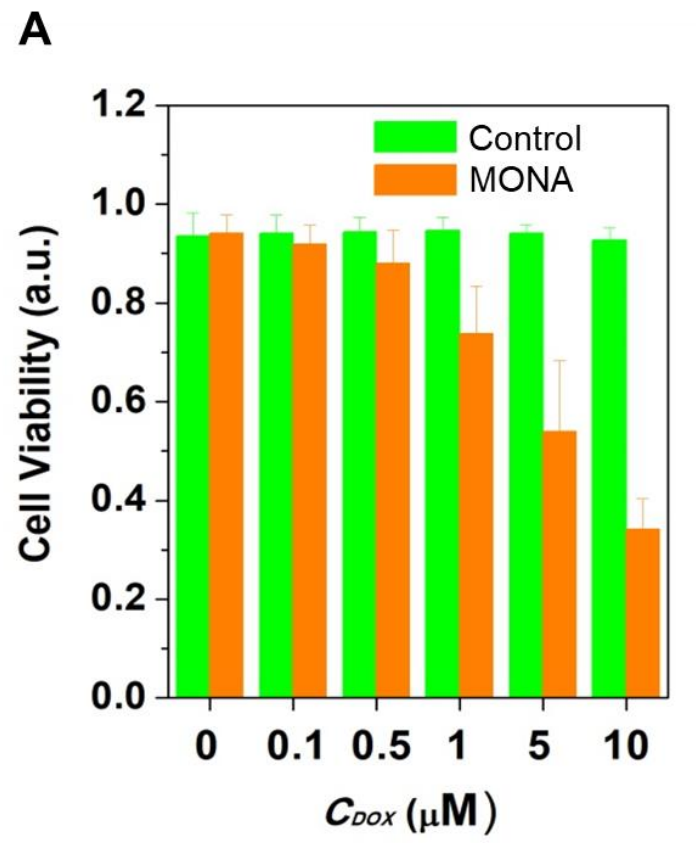

B

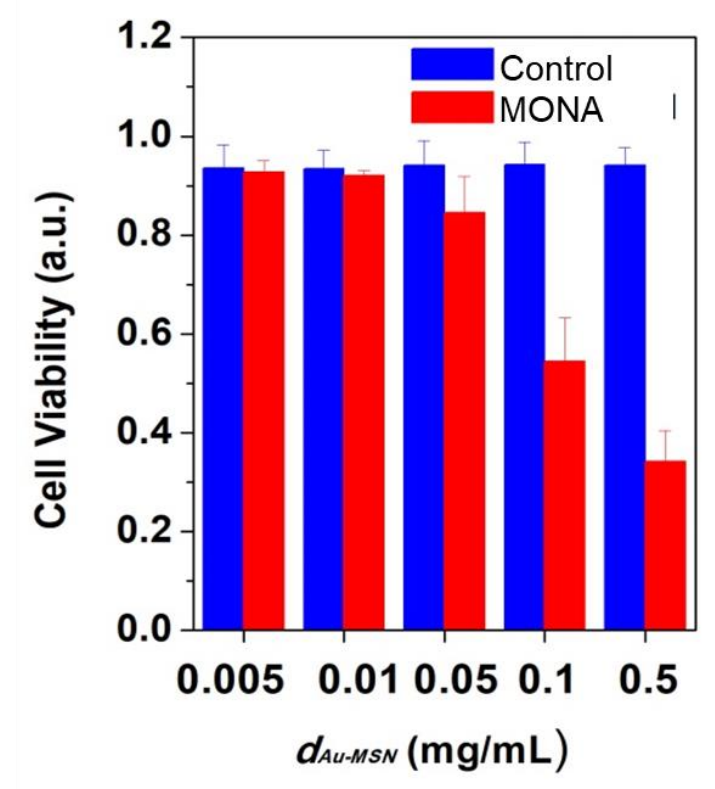

Figure S8. Cell viability test. (A) Cell viability of MCF-7 cells treated with MONA as a function of particle density at $C_{D O X}=1 \mu \mathrm{M}$ under light illumination and control (dark). (B) Cell viability of MCF-7 cells incubated with MONA-DOX for $72 \mathrm{~h}$, as a function of lorded DOX concentration under light illumination and control (dark). 


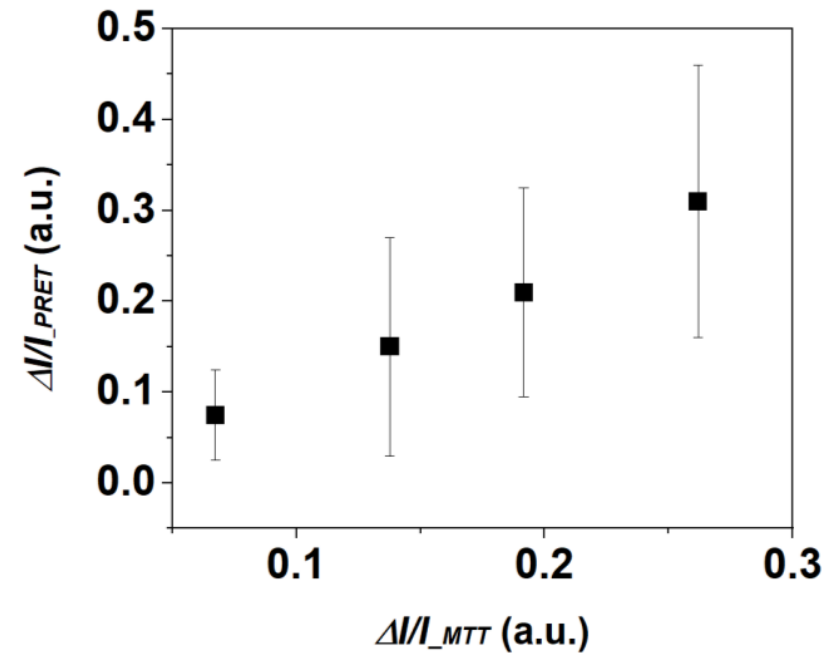

Figure S9. Comparison test. Linear regression between MTT assay and PRET data for the same samples treated with DOX-MONA ranging from 0.1 to $5 \mathrm{uM}\left(\mathrm{R}^{2}=9744\right)$. 
a

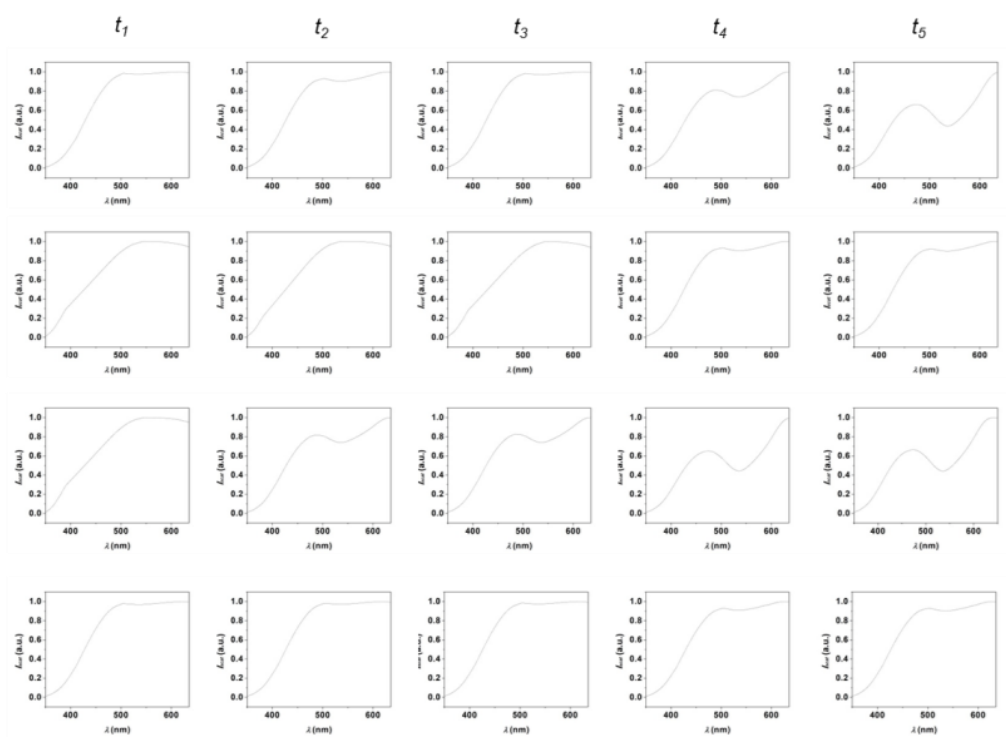

b
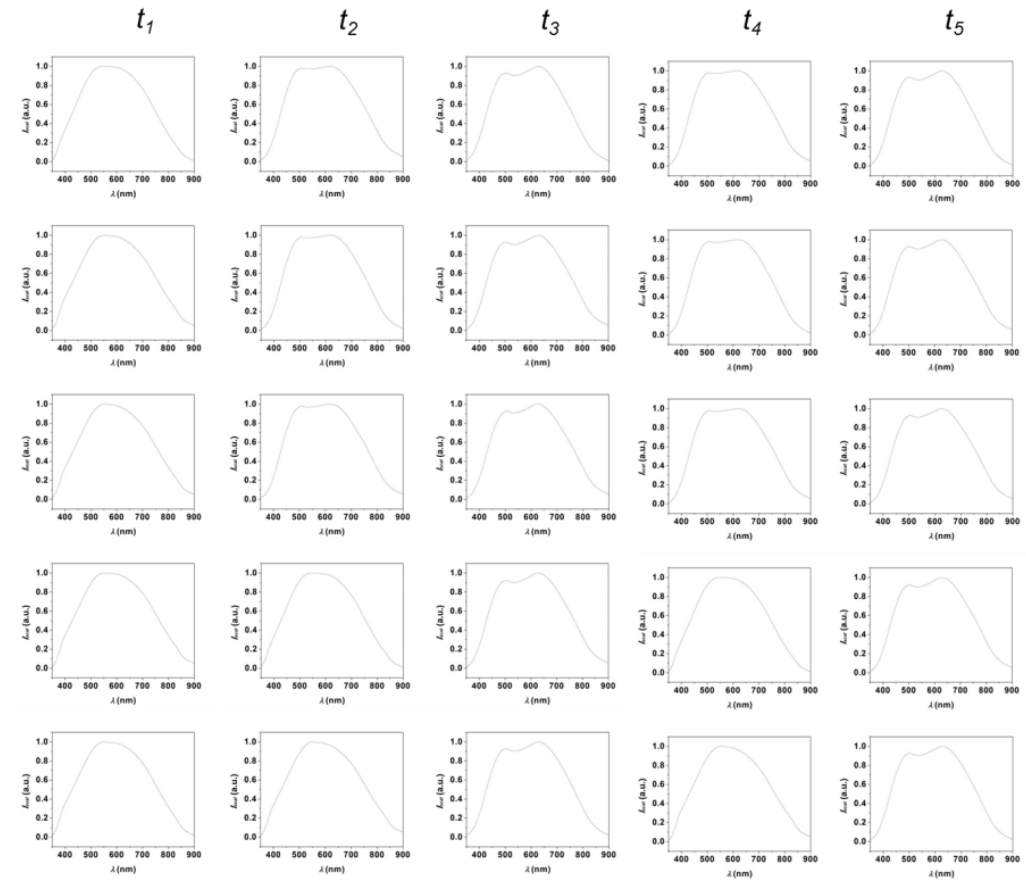

Figure S10. Dynamic intracellular PRET data; A, PRET signals as a function of time; $t_{1}=2, t_{2}$ $=4, t_{3}=8, t_{4}=16$, and $t_{5}=24 \mathrm{~h}$. B, PRET data of control sample as a function of time; $t_{1}=2, t_{2}=$ $4, t_{3}=8, t_{4}=16$, and $t_{5}=24 \mathrm{~h}$. 

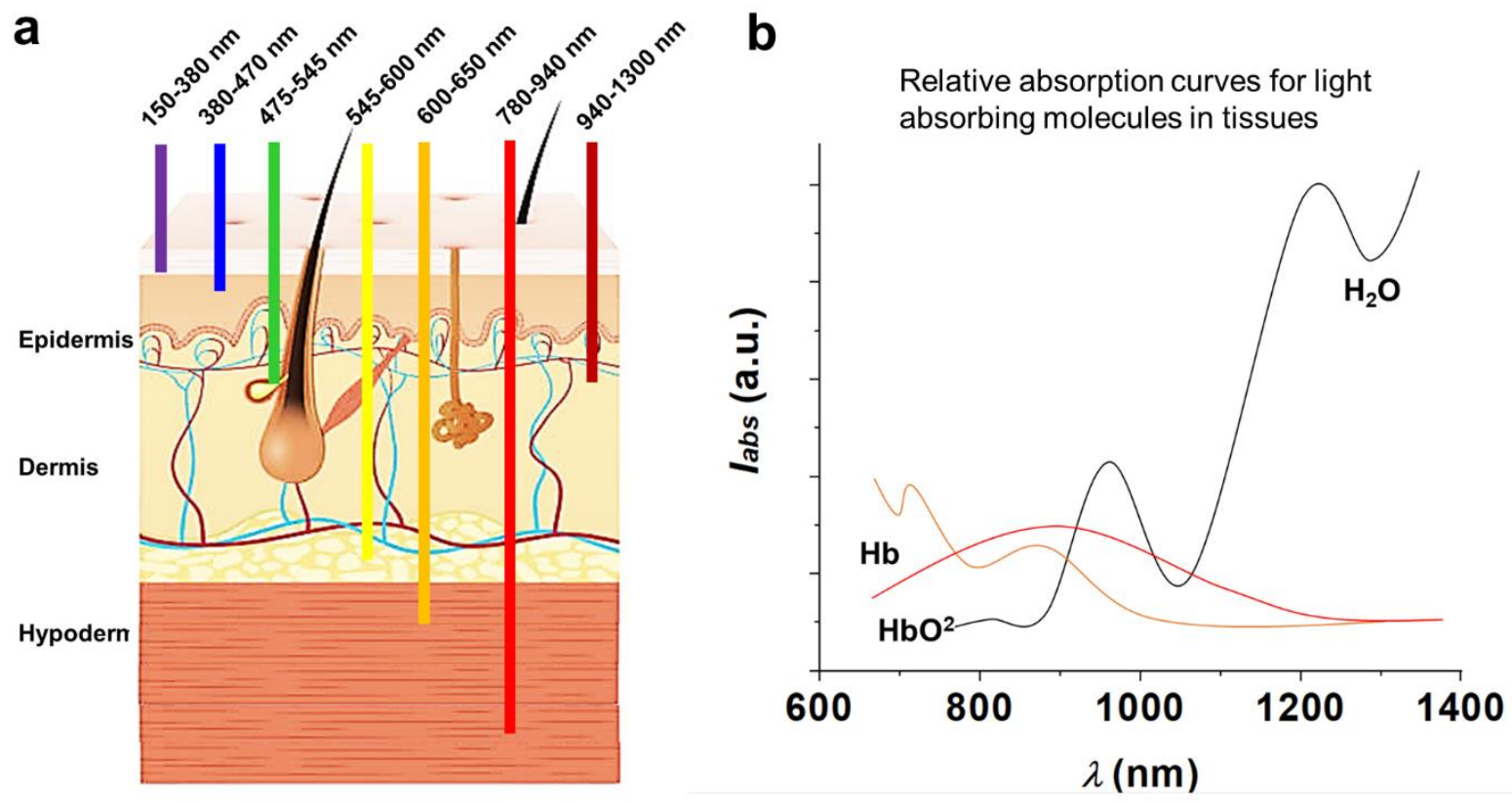

C

i)

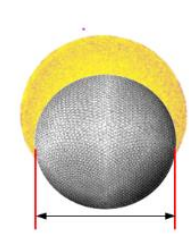

ii)

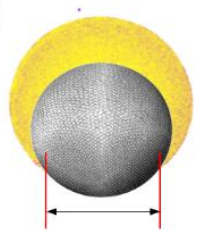

iii)

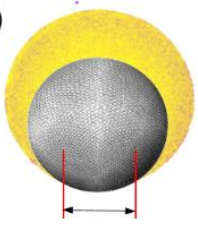

iv)

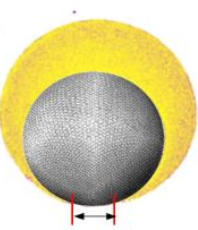

v)

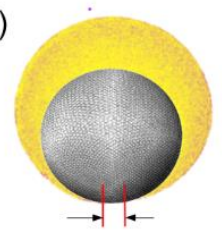

vi)

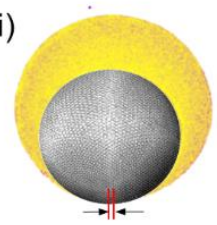

d

The NIR Wavelength Band for tissue in animal model

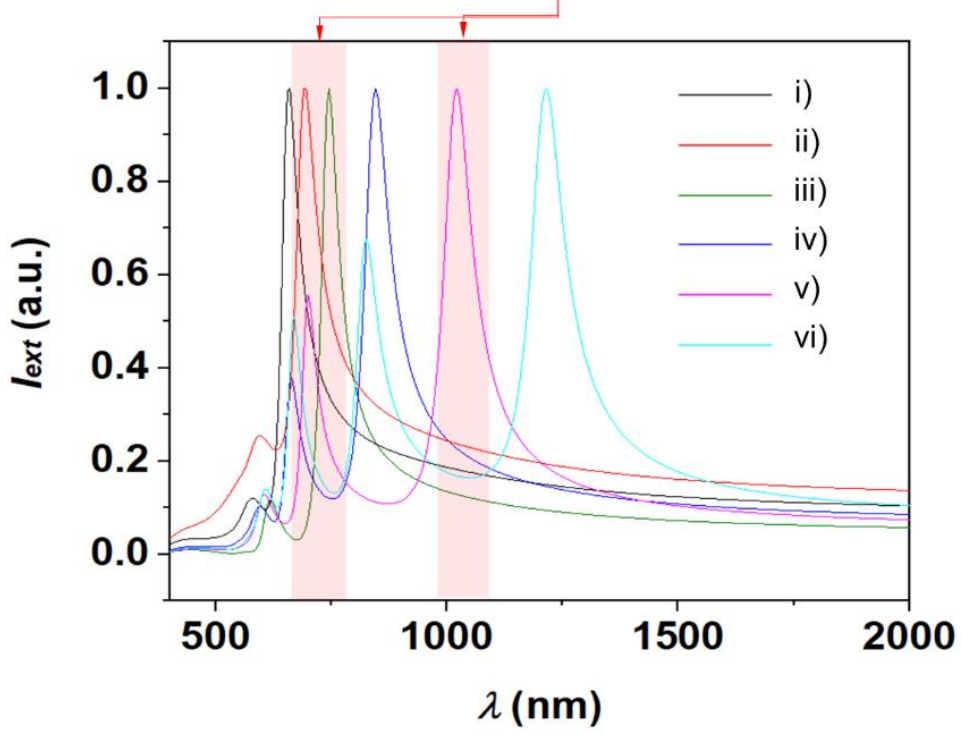

Figure S11. The spectral selectivity of MONA has potential for animal and tissue level studies. a) Schematic showing the change in the optical skin-depth at different wavelength of incident light in the tissue geometry, b) Optical windows in biological tissues. These plots of effective attenuation coefficient versus wavelength show that absorption and scattering from oxygenated blood, deoxygenated blood, skin and fatty tissue is lowest in either the first or second near-infrared window. c) schematic of MONA structures with edge-edge distances of i) 40, ii) 25, iii) 20, iv) 10 , v) 5, and vi) $2 \mathrm{~nm}$. d) Extinction spectra of MONA structures estimated with edge-edge distances of i) 40 , ii) 25 , iii) 20 , iv) 10 , v) 5 , and vi) $2 \mathrm{~nm}$. 